\title{
Modernité Tchicaya
}

\section{Daniel Delas}

\section{OpenEdition \\ Journals}

Édition électronique

URL : https://journals.openedition.org/coma/572

DOI : $10.4000 /$ coma.572

ISSN : 2275-1742

\section{Éditeur}

Institut des textes \& manuscrits modernes (ITEM)

\section{Référence électronique}

Daniel Delas, « Modernité Tchicaya », Continents manuscrits [En ligne], 5 | 2015, mis en ligne le 15 octobre 2015, consulté le 21 septembre 2021. URL : http://journals.openedition.org/coma/572 ; DOI https://doi.org/10.4000/coma.572

Ce document a été généré automatiquement le 21 septembre 2021.

\section{(c) (i) () $\Theta$}

Continents manuscrits - Génétique des textes littéraires - Afrique, Caraîbe, dispora est mis à disposition selon les termes de la licence Creative Commons Attribution - Pas d'Utilisation Commerciale - Pas de Modification 4.0 International. 


\title{
Modernité Tchicaya
}

\author{
Daniel Delas
}

\section{Modernité ?}

1 Comment définir le moderne dans le domaine littéraire? La réponse donnée par les dictionnaires: «actuel, de notre temps ou d'une époque récente" est trop lâche. J'emprunterai ma définition de la modernité à Henri Meschonnic dans son essai Modernité modernité $^{2}$ : «La modernité est un état naissant, indéfiniment naissant, du sujet, de son histoire, de son sens... la modernité est la vie, la faculté de présent... l'avenir du présent » (p. 9-13). On ne confondra pas le moderne avec l'actuel ou le contemporain qui désigne tout ce qui nous vient sans distinction; on peut même dire que le moderne s'oppose au contemporain, soumis aux effets de mode et désigne au contraire ce qui est irrécupérable par le contemporain ${ }_{2}$ parce que n'entrant dans aucun cadre connu. Il convient aussi de distinguer cette modernité, mise sous le signe du présent vécu et de l'invention d'un nouveau réel, de ce qui se configure à partir de l'histoire générale des idées et considère certains faits comme des tournants - la fin des avant-gardes, la disparition des postures de défi, la critique de la raison, etc. On est en effet alors dans l'histoire, dans la successivité historique, et non dans l'historicité, ce qui fait qu'un sujet, au moment même où il écrit, peint ou sculpte, est et vit dans l'histoire de son temps. Ce qui fait que Baudelaire a beau être chronologiquement contemporain de George Sand, il a vu dans son temps autre chose que ce qu'elle y a vu, la poésie des villes, la beauté du quotidien, de la "vie triviale ", pressentant ainsi la réalité de l'urbanisation comme phénomène majeur du XIx et du Xx ${ }^{e}$ siècle.

2 Dans cette perspective il faut en venir à considérer l'existence de modernités au pluriel, se succédant sans nécessaire continuité. S'il y a une certaine continuité de la modernité Baudelaire à la modernité Rimbaud du point de vue de l'histoire littéraire, il n'y en a pas entre la naissance de sujets à chaque fois nouveaux en tant que tels. Quelle continuité entre une modernité Joyce et une modernité Pessoa, une modernité Michaux et une modernité Kourouma? À un niveau élevé de généralité comparatiste l'histoire littéraire peut bien en établir un semblant, mais n'est-ce pas une acrobatie verbale puisque 
chacune de ces modernités, située dans un combat pour le sens du monde, ne lui donne pourtant pas le même sens? La vision eschatologique de la succession des modernités comme une marche vers une postmodernité radicale faite de perte définitive du sens est une idée à la mode mais superficielle. Parce que cette perte du sens, ce sentiment de perte du sens, fait toujours partie du sens et « en est même le témoin le plus intense » (p. 266). D'ailleurs le présent ne peut pas s'observer de l'extérieur. La question du sens ne peut jamais en effet devenir vaine, pas plus que celle du sujet, comme le dit justement encore Meschonnic: "Le sens est le recommencement du sens. Ce qui recommence à chaque sujet. Il y a nécessairement de l'inconnu dans le sens. Au moment de se faire. » (p. 269)

3 C'est à partir de cette idée du moderne que je voudrais considérer la modernité Tchicaya U Tam'Si. Qu'en est-il de cette modernité en son temps et dans son histoire face à telle autre? Tchicaya, Senghor, Camara Laye, Mongo Beti, pour ne citer que quelques noms, sont plus ou moins des contemporains et ont parfois habité les mêmes lieux, se rencontrant éventuellement, l'histoire de leur temps les a informés en principe également et pourtant ils ont produit des œuvres différentes, voire contrastées. Pourquoi ? Pourquoi surtout, avec le recul, certaines nous paraissent-elles plus riches, plus « en avance », plus... modernes ? Parce qu'ils ont été des individus-auteurs avec des personnalités plus fortes? La réponse est faible, on le sait depuis la critique du beuvisme. Ce qui ne veut pas dire qu'il n'y ait pas d'enseignements à tirer de tel ou tel fait biographique mais qu'il faut le faire à partir de cette inventivité sans cesse renaissante dont Meschonnic parlait. Ne pas tant creuser la psychologie de l'auteur individuel mais mont(r)er certains détails biographiques dans leur historicité propre.

\section{Quelques anecdotèmes}

Commençons par quelques détails biographiques qui peuvent sembler microscopiques mais sont, ne serait-ce qu'en tant que points de départ situés dans un vécu, chargés de sens.

5 Le premier trait spécifique de la modernité Tchicaya à pouvoir être mis en relation avec ce que fut l'individu est son rejet de la culture lettrée française. Mauvais élève à la scolarité cahotante, révolté contre la tutelle paternelle, mal à l'aise dans sa famille, souffrant d'un handicap physique, Tchicaya se met progressivement en marge, disons dans une certaine marge : il refuse de profiter pleinement de la position de son père et exerce de "petits boulots", comme on dit aujourd'hui. En rupture avec son milieu d'origine et avec la culture scolaire officielle. Notée par tous les critiques, la référence à Rimbaud, lui-même marginal et peu reconnu à son époque par les manuels scolaires, est aussi existentielle. Aimée Gnali, qui a suivi son cousin dans une part importante de sa scolarité et de sa vie, rappelle utilement pourquoi il s'est senti mal à l'aise au lycée d'Orléans, préférant la peinture à la littérature et n'empruntant le titre de son premier recueil Le Mauvais sang à Rimbaud, dont la trajectoire rebelle le séduisait, que parce que cette expression correspondait à une expression meng'm-bi (en vili) ou makila mabe (en lingala). Autrement dit, s'il veut bien accepter la filiation Rimbaud - et ce sera la seule avouée -, filiation française et littéraire, c'est pour la subvertir aussitôt en la farcissant d'africanité. C'est dans la ligne de ce refus de la culture lettrée qu'il ne se reconnaitra aucune parenté littéraire avec Senghor, Césaire ou même Damas, les pères de la négritude, si omniprésents dans les années 1950. 
6 Il a par la suite toujours persisté dans cette attitude de rejet et l'on se souvient qu'il n'a pas voulu, lors d'une réédition, reprendre la préface que Senghor avait accepté de donner à son recueil Épitomé en 1962. Pourquoi ? Sans doute pour refuser l'embrigadement dans les rangs de la négritude que le poète sénégalais imposait à tous ceux dont il faisait l'éloge mais, plus profondément, parce que le monde lettré, voire érudit, de l'agrégé de grammaire n'était pas le sien et que son projet poétique était radicalement autre.

7 Une anecdote qu'a racontée Tahar Bekri fera figure de second anecdotème, dans le sens que lui donne Barthes, quand il relate que, lors d'un voyage qu'ils firent en Haïti, Tchicaya refusa d'aller visiter la citadelle du roi Christophe. « Tchicaya ne désirait pas venir parce qu'il n'avait rien à faire avec les pierres mortes, me disait-il, et qu'il préférait aller à la rencontre des intellectuels de Jacmel et du génie du peuple 3 .» On comprend mieux pourquoi, dans la ligne de cette attitude anticonformiste, Tchicaya se définit comme un homme de son temps, s'intéressant au jardinage, au bricolage, aux voitures, bref à tout ce qui est censé ne pas devoir intéresser un poète. Il n'est ni clerc ni lettré, ni instituteur ni professeur, ni congolais ni français mais «congaulois", comme il l'a dit une fois, recourant à un jeu de mots peu distingué, voire de mauvais goût, tel que s'autorisent à en faire des gens « sans qualités ». Jeux de mots qu'il multipliera avec délectation (p. ex. lutte des crasses / lutte des classes) dans son œuvre poétique comme autant de provocations, comme pour dire : Je ne suis pas des vôtres, je ne suis ni « pohète » ni prophète.

8 On pourrait rappeler bien d'autres petits détails biographiques corroborant cette posture de l'individu Tchicaya qui iraient dans le même sens d'une volonté d'être moderne, d'assumer son temps et son présent à sa manière, sans allégeance culturelle, comme un homme simple et compliqué à la fois, sans autre " posture » que de récuser tous les rôles que la bienséance politiquement correcte lui propose. Cette volonté a certes un coût psychologique, elle implique une attitude volontiers provocante, une réactivité parfois brutale, le risque de dérouter ses meilleurs amis. Cette posture peut mener au repli sur soi ou à des dérapages paranoïdes graves, comme Céline en a donné l'exemple. Elle ne signifie pas que Tchicaya n'avait aucune culture mais qu'il s'est mis dans une posture de refus de la culture héritée ou dominante, africaine ou française, se condamnant ainsi à s'assumer comme moderne. Pari risqué, hautement périlleux. La seule comparaison qu'on peut faire, me semble-t-il, est avec Césaire, qui, pour écrire le Cahier d'un retour au pays natal, comprend qu'il lui faut rejeter l'héritage culturel occidental et ses « richesses pérégrines ", la différence étant que Césaire pour faire ce terrible travail trouve dans le projet surréaliste un allié qui lui apporte son aide, tandis que, vingt ou trente ans plus tard, Tchicaya entre seul dans l'aventure de l'écriture poétique sans aucun allié littéraire. N'est-ce pas ce qui s'entend dans ce tardif poème inédit :

Je passai par six portes

La septième irréelle

Sur fenaisons faites

Large seuil et silence

\section{La « parenthèse de Kin »}

9 L'expérience que Tchicaya fait de la marche de l'histoire d'août à octobre 1960 à Kinshasa est essentielle. «En juin 1960, raconte le poète, un coup de tonnerre, c'est la réelle indépendance de l'Afrique qui commence. (...) En juin 1960 commence pour moi une geste magnifique, celle de Patrice Lumumba. Et je suis Croisé. Je pars. Je pars sans armes ni bagages avec une passion contenue et tonitruante. » Mais peu avant de partir, il apprend 
à Bruxelles la mutinerie du camp Hardy et dans le train qui le ramène à Paris, le 7 juillet, il écrit «La Conga des mutins", qui ne fut publié qu'en 1978 et est un des textes qu'il prenait plaisir à lire en public. La «parenthèse de Kin» est un point de bascule capital dans l'expérience du monde de Tchicaya et dans l'affirmation de son écriture propre, déterminant le moment où il va pleinement assumer une modernité, certes déjà présente dans sa poésie mais qui va prendre toute sa dimension tragique-sarcastique.

\section{Un poème moderne : « La Mise à mort »} plus tard députée et ministre de la Culture), est daté à la fin du poème de mars 1977 ; il est divisé en strophes (S) inégales numérotées de 1 à 18. Il sera publié en 1978 par Présence Africaine, avec « La Conga des mutins » (daté de juillet 1960), dans Le Pain ou la Cendre avec la réédition de Le Ventre, publié pour la première fois en 1964. Même si Tchicaya a donné quelques poèmes isolés après 1977 à des journaux ou revues, ce poème peut être considéré comme le dernier poème du dernier recueil publié de Tchicaya U Tam'si, et possède de ce fait une indéniable valeur testamentaire, par son contenu même.

rhétorique ou la stylistique sont peu opérants, sitôt la première lecture achevée. Pourrat-on se contenter de parler du jeu des temps ou des sonorités, de la composition symphonique, de la thématique satirique ou tragique dominantes? Non, on le sent, s'il est vrai que l'important dans un poème centré sur le vécu est dans le mouvement vivant, dans le rythme d'ensemble qui donne vie et sens au texte. On voudrait pourtant éviter, par découragement devant les difficultés d'une lecture critique attentive au détail, de verser dans les généralités grandiloquentes, à la manière de ce critique empathique qui écrit, certainement avec les meilleures intentions du monde : «Du Mauvais sang, qui date de 1955, aux Notes de veille de 1977, le chemin de Tchicaya a traversé un hermétisme fait du mystère de sa race et de sa terre pour arriver à une angoisse métaphysique à l'exemplaire lisibilitét. »

arle ici ? C'est un texte à la première personne (commençant par « Je » et dialoguant avec un autre lui-même « Tu»), une sorte d'adieu à la vie, à une vie difficile, nous en avons évoqué quelques aspects, tendue à l'extrême entre orgueil et humilité, humanité congolaise-africaine et humanité française-occidentale, entre désir de reconnaissance et rejet des modèles usuels :

Je pourrai dire merde à ma vie

Et m'en aller l'âme en écharde

14 Un poème d'adieu, encore plaintif sans doute «l'âme en écharde ", mais désormais inébranlable dans ses refus « merde ». Adieu qui résonnera longuement en final:

je m'en vais

adieu...

adieu...

adieu...

adieu!

$15 \mathrm{Au}$ cours de ce monologue, le poète passe souvent au "Tu», se dédoublant et s'interpellant (S. 2) et (S. 3), avant de revenir au «Je ». 
Une sorte de tournant se produit en (S. 11). Les dix premières strophes sont une sorte de soliloque amer, un commentaire désabusé d'un mort encore vivant ou d'un vivant déjà mort qui commente son vieillissement (S.1), ses derniers actes en ce bas monde « Tu danses la dernière fois » (S. 2), se souvient de ce qu'il a été dans sa jeunesse enthousiaste (S. 4), de ses "soliloques vineux» (S.5), qui parle d'amis disparus, se perd dans les parenthèses de ses propres souvenirs :

Où en étais-je je ferme la parenthèse. Où en étais-je ?... Ah félicité

Le mot à ne pas dire devant qui la mort

Aurait pu être cela à condition que... Oh ma

Tête, mon dieu, je ne sais plus.

17 s'émeut attendri au souvenir de son entreprise presque joyeuse, jubilatoire, de destruction et s'anime soudain (S. 6), en énonçant le programme iconoclaste qui a été le sien et qui l'est plus que jamais :

Souiller le parler politique d'immondices

Afin que les peuples majeurs se rebellent

En souillant leur mort de sang perlé

D'hydromel, de jus de canne à sucre,

D'huile de noix de cajou pour l'extrême-

Onction et que bénit soit le prophète...

18 On note ici la reprise en anaphore du mot souiller. Le poète s'anime, martèle ses anathèmes contre l'universelle langue de bois menteuse qui brouille tous les discours, sa hargne se marque par l'enfilade des noms de produits salissants qui lui viennent à l'esprit, terminant par la pirouette sardonique d'une formule rituelle religieuse. Il retrouve ce faisant, sans l'observer vraiment, une certaine isométrie, simplement sous la pression de l'efficacité rythmique

19 Sa prose poisseuse me fait penser - moi lecteur / lui auteur ? - aux vers vengeurs de Césaire ${ }^{5}$, le jeune Césaire du Cahier d'un retour au pays natal dont l'écriture faisait passer un souffle "péléen» sur le vieux monde blanc et exprimait ses espérances dans la renaissance des noirs :

Et ces têtards en moi éclos de mon ascendance prodigieuse !

Ceux qui n'ont inventé ni la poudre ni la boussole

Ceux qui n'ont jamais su dompter la vapeur ni l'électricité

Ceux qui n'ont exploré ni les mers ni le ciel

Mais ils en savent en ses moindres recoins le pays de souffrance

20 Lui aussi, Tchicaya, il a cherché à magnétiser son peuple mais il est aujourd'hui plus proche du Césaire vieillissant de Moi laminaire ${ }^{6}$ :

Moi qui rêvais autrefois d'une écriture belle de rage !

Crevasse j'aurai tenté

21 À la différence du vieux maître qui «enrage de n'avoir pas la vertu qui renonce » et qui clama toujours la nécessité d'avoir « la force de regarder demain », lui Tchicaya renonce et cherche la mort, quelque plein de rage qu'il se sente encore. Pourquoi ? Peut-être d'abord parce que la mise à mort de Patrice Lumumba a été vécue comme sa propre mise à mort? ${ }^{7}$ il y a eu «truchement d'embolie » (S. 18), «mise à mort du soleil» (S. 18), l'Homme a perdu le combat du rêve «Patrice tu rêvais Patrice tu ne rêvais pas " (S. 17). Ensuite parce que, dans la droite ligne de son œuvre poétique, elle est le « pouce obscur du destin $»^{8}$ qui lui dit qu'il est temps de partir, puisqu'on a tenté, dans l'excès et la naïveté certes mais sincèrement. 
mais mourir d'un excès d'atomes

n'a jamais fait de mal à personne

et nous prions dieu qu'il veuille bien nous

pardonner du péché d'impiété juvénile (S. 7) à l'histoire de son Congo qui soudain fait irruption avec l'évocation exactement chiffrée de l'exécution sommaire du cardinal Biayenda et des anciens présidents Marien Ngouabi et Massembat-Débat en 1977. Tchicaya n'était pas sur place, mais il sait ce qui s'est passé et, en écrivant, vit les événements politiques avec une intensité visuelle forte. C'est de vraie boue et de vrai sang qu'il nous parle. Il voit la haute silhouette de la cathédrale assister impassible aux massacres :

et à nouveau Sainte Anne témoin impavide sans anathème va d'une homélie douce 
tragiques dans le détail (S. 15) :

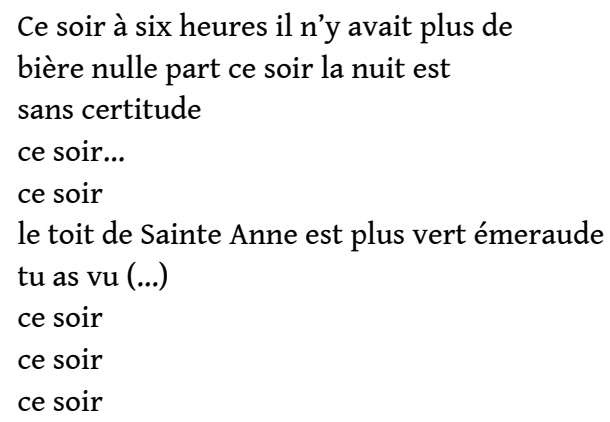

La mort du poète accompagne la mort du cardinal et des deux hommes d'État, elle est celle de la "liberté nègre", de la "liberté congolaise». Revient pour le dire le mot écharde qui, on s'en souvient, avait ouvert le poème « l'âme en écharde » sur un mode un peu goguenard («l'âme en écharde» consonnant avec la mort en écharpe) mais qui désormais associé à la « liberté » ne prête plus à plaisanter « s'étrangle[r] d'agonie » :

La liberté congolaise est mon écharde au

cœur comme moi après Kin le fleuve

s'étrangle d'agonie

Remonte alors en rêve du fond de la mémoire le souvenir du rêve de Lumumba qui l'a mené au supplice. Pour dire « Mort aux rêveurs! Lumumba / Tchicaya même défaite ! ».

Et pourtant Lumumba le disait le congo

c'était la splendeur la merveille nec plus ultra

paré de jacinthes d'eau on ne saurait être

plus coquet que ce fleuve. Anne lange-le

d'humus. Ah le nègre vous verrez prophétisait-il

Patrice tu rêvais Patrice tu ne rêvais pas.

Et pourtant libre il se militarise instaure la paix

Concentrationnaire avec miradors et

Fosses d'aisance pour diarrhéiques et

Sommeilleux.

Avant de s'achever dans l'ultime adieu évoqué à l'instant.

\section{Modernité poétique}

31 Ce qui est moderne dans ce poème dont on a tenté d'éclairer les flux et contre-flux, ce n'est évidemment ni la douleur existentielle de l'écrivain ni la dimension tragique de l'histoire africaine, ces données sont tout simplement humaines et il est juste à propos de Tchicaya de parler de la dimension universellement humaine de la douleur d'un homme assumant son histoire sans vouloir la dissocier de celle de son pays. C'est dans son écriture du vécu que la modernité de Tchicaya se manifeste.

D'abord par le suivi sans trève des moindres mouvements de sa pensée, comme dans une sorte de fusionnalité constante entre le dire et le penser. Le rythme tchicayen - celui en tout cas de son écriture tardive - n'est pas contrôlé par une intentionnalité esthétique qui disposerait savamment les échos sonores, les harmonies périodiques, les parallélismes et leur rupture, à la manière d'un Valéry ou d'un Senghor, c'est le rythme d'une pensée en quête de son expression, dans le temps même de son expression, au plus présent du moment présent. Ce que Meschonnic, cité en introduction, appelle «la faculté du 
présent ». Sa syntaxe n'est plus gouvernée par une ordonnance phrastique rigide, par cette ossature qui assure la prise de pouvoir de celui qui écrit. Tchicaya se déprend de tout pouvoir discursif, il écrit à l'écoute du vivant dans ce qu'il a de plus éphémère, de plus liquide :

bois par-dessus chaque mort si tu veux qu'il fleurisse

dans l'oreille qui t'écoute. L'oreille qui m'écoute?

quelle oreille?

Cette belle et étrange expression fleurir dans l'oreille qui t'écoute est à prendre au pied de la lettre : l'écoute, dans et par le langage le plus réduit à sa simple expression, garantit seule la vérité et la sincérité de l'émotion. Sans la surcharge inutile et menteuse d'une organisation discursive savamment élaborée qui ne pourra que trahir le vivant. Dans une humilité qui est la seule garantie d'humanité possible.

Ensuite, dans l'assomption du sale et de l'horrible, sans la surenchère verbale gratuite et burlesque qu'on pourrait trouver dans certains textes céliniens, selon un rythme qui vient du ventre. Sony Labou Tansi, ce «fils » aimé mais souvent brusqué, disait dans une lettre à Françoise Ligier écrite en 1977, soit l'année même de l'écriture de ce poème ${ }^{10}$, qu'il aimait ce qu'écrivait Tchicaya U Tam'Si parce que " Ça a[vait] du ventre. Pas comme ces types qui écrivent comme des professeurs. » En quoi ce passage a-t-il « du ventre »?

Aucune lumière n'est une / onction pour l'extrême survie plantigrade / opiacée, cucurbitacée ou tout simplement / nègre nègre incolore à quelques kilotonnes / près de douleur nègre la mauvaise bête / puante réfractaire à l'idée de la nudité / du sang à la bouche ouverte/ commanditaire de vaginites pour pubis en quarantaine / dans quelque lazaret en bordure de mer / tiède paludée (17)

Parce qu'il refuse la fausse lumière de l'onction lettrée et se penche à l'écoute de la vie animale de l'homme "plantigrade » - qu'il soit nègre si l'on veut, mais nègre sans négritude, "nègre incolore", sans se boucher les oreilles ni le nez, une humanité " puante », couverte de « sang ", atteinte de maladies sexuelles ("vaginites »), en attente de la fin dans quelque « lazaret ». Les deux derniers mots, tiède paludée, peuvent-ils se lire comme un apaisement? Ce serait se rassurer à bon compte, en lecteur qui cherche désespérément jusqu'à le fantasmer le moindre rai de lumière ${ }^{11}$. Le palud, c'est toujours le marécage pestilentiel, celui qui, chez Césaire, asphyxie nombre des poèmes de Moi laminaire. Par exemple dans « La condition-mangrove» :

Noire la mangrove reste un miroir.

Aussi une mangeoire.

La mangrove broie-tapie à part

La mangrove respire. Méphitique. Vasard.

On ne prétendra pas juger de toute l'œuvre poétique de Tchicaya à partir de la lecture de ce poème Mise à mort mais c'est bien dans et par ce que dit ici cette écriture que le meilleur d'une modernité spécifique (africaine ?) se dit, avec souffrance et dérision. 


\section{NOTES}

2. Éditions Verdier, 1988, rééd. Folio Essais Gallimard 1993, 316 pages.

3. «Tchicaya U Tam'si comme un arbre à pain », Cultures Sud, « Tchicaya Passion », $\mathrm{n}^{\circ} 171$, coord. par Daniel Delas et Boniface Mongo-Mboussa, p. 78.

4. Pierre-Jean Rémy, "La mort d'un poète nous laisse ses mots ", in Nino Chiappano (dir.), Tchicaya notre ami, AAFU / ACCT, Paris, 19-25, p. 20.

5. Cahier d'un retour au pays natal, in Aimé Césaire, La Poésie, Seuil, Paris, 1994, p. 40.

6. Moi laminaire, «Crevasses », op.cit. p. 447.

7. D'autant que la mort du père de Gérald, ce père si fortement présent, l'a accompagnée.

8. Pour reprendre la belle expression de l'écrivain rebelle américain Hunter S. Thompson (1937-2005), si proche de Tchicaya dans ses anathèmes contre l'American Dream et les politiciens corrompus.

9. On pense par exemple au poème «Le Contempteur » d'Épitomé, Pierre-Jean Oswald, Honfleur, 1970, p. 61-65.

10. L'Atelier de Sony Labou Tansi - tome 1 - Correspondance, édition établie par Nicolas MartinGranel et Greta Rodriguez-Antoniotti, Revue Noire, Paris, 2005, p. 212.

11. Comment écrivait Tchicaya? Il y aurait de nombreux textes de lui à citer mais puisque j'ai commencé à faire un rapprochement avec Hunter S. Thompson, je poursuis, persuadé qu'il y a quelque chose de commun entre ces deux écrivains qui s'ignoraient totalement. Un ami de Thompson le décrit ainsi en plein travail d'écriture, dans les seventies': "J'ai vu comment il s'asseyait à la table avec une Selectric devant lui, les coudes bien sur les côtés, et puis il avait cette espèce de décharge électrique et il se mettait à taper. Une phrase, puis il attendait de nouveau, les bras en dehors, il avait une nouvelle décharge et il tapait une autre phrase. À l'observer j'ai commencé à comprendre que ce qu'il essayait de faire, c'était de contourner les points de vue pesants, les idées reçues, les clichés, tout ça, pour parvenir à quelque chose davantage lié à son inconscient et à sa perception immédiate des choses. Il voulait en quelque sorte sortir la phrase, avant que quoi que ce soit n'interfère avec elle en fait de convention ou de préconception. » (cité dans William McKeen, Hunter S. Thompson journaliste et hors-la-loi, Tristram, 2009-2010, p. 270)

1. Batouala : véritable roman nègre, de René Maran, est paru chez Albin Michel en 1921 à Paris (prix Goncourt 1921).

\section{RÉSUMÉS}

La notion de modernité permet-elle de mieux comprendre les lignes de force qui traversent en profondeur les littératures francophones depuis qu'elles se sont affirmées il y aura bientôt un siècle, si on fait partir leur histoire de la parution de Batouala ${ }^{1}$ (1921) ? Et de mieux réfléchir à une approche de l'œuvre poétique de Tchicaya U Tam'Si? 
INDEX

Mots-clés : Congo, Tchicaya U Tam'Si, Henri Meschonnic, Aimé Césaire, Sony Labou Tansi, Patrice Lumumba, modernité

\section{AUTEUR}

DANIEL DELAS

Professeur émérite, Université de Cergy-Pontoise 